\title{
Topical Steroids and Elimination Diets for EoE: Happier Together?
}

\author{
Carla M. Davis ${ }^{1}$
}

Published online: 19 April 2018

(c) Springer Science+Business Media, LLC, part of Springer Nature 2018

Eosinophilic esophagitis (EoE) is an inflammatory esophageal disease triggered by ingestion of food allergens, resulting in symptoms such as heartburn, abdominal pain, nausea, vomiting, and dysphagia. Symptomatic, endoscopic, and histologic remission is achieved after treatment with either topical steroids or dietary elimination of the top 4 or 6 most causative foods (cow's milk, egg, soy, wheat, nuts, and seafood) [1]. There has been limited study of the efficacy of the combination of these two approaches [2]. Moreover, there are many questions and controversies regarding a combination therapeutic approach.

Questions surrounding practical management of EoE in the office setting are several. Does treatment with topical steroids at the time of diagnosis hasten symptomatic improvement while patients establish a new diet? Does the dose of topical steroid or the number of food avoidances affect the speed of remission? Can these two therapies combined induce remission faster than single therapy? Are alternating therapies or therapeutic "holidays" valid approaches to therapy?

In this issue of Digestive Diseases and Sciences, Reed et al. [3] report on the results of a retrospective cohort study designed to determine efficacy and compliance in pediatric EoE patients receiving sequential treatment with a combination of topical steroids and a 2 food elimination diet (2FED) of cow's milk and soy followed by treatment with 2FED alone. Each therapy was administered for 3 months followed by biopsy. The combined approach resulted in symptomatic, endoscopic, and histologic improvement. When steroid treatment was discontinued, eosinophilic inflammation reoccurred, although symptomatic and endoscopic improvements persisted through the final clinical and endoscopic evaluations.

Carla M. Davis

carlad@bcm.edu

1 Section of Immunology, Allergy and Rheumatology, Texas Children's Hospital Food Allergy Program, Baylor College of Medicine, Texas Children's Hospital, 1102 Bates Avenue, MC 330.01, Houston, TX 77030, USA
Strengths of this study include the novelty of the approach (2FED of cow's milk and soy simultaneously with budesonide) and the comprehensive assessment, including preand post-treatment weights. A report from the same institution documented the relationship between decreased body mass index and EoE diagnosis [4]. Anecdotally, clinicians observe patients treated with dietary elimination who do not consume as many calories after diet initiation compared to before initiation of the diet therapy. These patients continue to have poor weight gain. Reed et al. do not report a significant weight loss with 3 months of 2FED therapy, which is particularly reassuring in patients who initially have a low body mass index, since there is a concern for further weight loss with diet elimination [3].

The "elephant in the room" in most studies of diet elimination therapy in EoE is the lack of assessment of patient compliance to therapy. Obviously, this is a major confounder since it is well known that some patients have inflammatory disease triggered by even trace amounts of allergenic food exposure. This study addresses this issue, documenting compliance in approximately three-fourths of the patients through parental report and physician documentation of compliance in the medical record. It is commendable for the authors to address this, even though parental reporting is frequently inaccurate. Superior methods of compliance assessment are needed in future studies, as it is an important aspect of dietary elimination therapy. Retrospective noncompliance was associated with increased dysphagia and a statistical trend for increased post-treatment eosinophil counts/high powered field (hpf). More studies should attempt to assess compliance to therapy in order to validate the reported results, especially if years have passed since therapy initiation.

It is interesting that there were no significant differences between responders to combination therapy in the partial/ complete compliance groups compared to the inadequate compliance group, even though the treatment effect trended toward less response with inadequate compliance. This trend supports the hypothesis that patients who are less adherent to dietary restriction should be treated with corticosteroids. 
An unexplored issue is the comparative efficacy of combination therapy with corticosteroids and food elimination versus single therapy with topical corticosteroids. It is additive or synergistic?

The amount of food allergen ingestion necessary to trigger inflammation in EoE is typically described as miniscule [5]. Many expert physicians recommend avoidance of foods with trace amounts of allergen, including cross-contact based on anecdotal experience, whereas others allow patients to only avoid foods with large amounts of the culprit allergen. The Reed study would suggest that the strict avoidance requirement may not apply to all EoE patients. Foods with the allergen as one of the top ingredients would be avoided in this case, rather than foods with the allergen as the last listed of 30 ingredients on the nutrition label, for instance. Comparative assessments of the effect of compliance with food elimination diets on outcomes in large EoE patient populations are needed to clarify this issue.

The limitations of this study include the small number of patients, the retrospective design, and the assessment of histology by a single pathologist, rather than 2 blinded pathologists [6]. Furthermore, the choice of soy as one of the two foods in the 2FED was not a choice most likely to induce remission in EoE patients. Cow's milk exposure is the most common cause of esophageal inflammation in EoE, and recent studies with 4 food elimination diets (4FED) [7] and 6 food elimination diets (6FED) [8] have reported milk and wheat as more likely triggers compared with soy. Soy only triggered inflammation in approximately $20 \%$ of EoE patients compared with over $80 \%$ with wheat. Therefore, cow's milk and wheat should be considered as the most allergenic foods to avoid in a 2FED. Choosing milk and wheat avoidance may have produced more favorable outcomes, including less or no rebound after steroid discontinuation.

Hindrances to achievement of clinical, endoscopic, and histologic response in EoE abound, including financial constraints, since a specific topical steroid may not be reimbursed by insurance and diet avoidance of the top 4 or 6 foods can be costly [9]. This report of the efficacy of combined steroid treatment and dietary avoidance expands the options for treatment. Since rebound occurred with abrupt discontinuation of corticosteroids, is it possible that a gradual approach to discontinuation of corticosteroids would be an alternative strategy to minimize this rebound effect? This issue should be explored in future studies.

Combination therapy in EoE with topical budesonide mixed with an amino acid powder formulation and 2FED excluding cow's milk and soy is an attractive, practical treatment plan in patients having difficulty with multiple food avoidances. This approach is effective in inducing clinical, histologic, and endoscopic remission. Quality of life may also be improved with this novel therapeutic approach, since this measure is improved with less dietary restrictions [10]. Further assessment of the combination regimen should be considered for prospective multicenter trials. Future directions for refinement of this approach include the assessment of gradual weaning of corticosteroids after combination therapy, the use of cow's milk and wheat as a 2FED, and the determination of the relative effect of steroid therapy versus diet elimination. The options for EoE treatment are no longer singular in focus. Combination therapy brings flexibility and options for patients with this chronic lifelong disease.

\section{References}

1. Dellon ES, Gonsalves N, Hirano I, et al. ACG clinical guideline: evidenced based approach to the diagnosis and management of esophageal eosinophilia and eosinophilic esophagitis (EoE). Am J Gastroenterol. 2013;108:679-692.

2. Constantine G, Seth N, Chokshi N, et al. Combination steroid and test-based food elimination for eosinophilic esophagitis: a retrospective analysis. J Pediatr Gastroenterol Nutr. 2017;64:933-938.

3. Reed CC, Safta AM, Qasem S, Almond MA, Dellon ES, Jensen ET. Combined and alternating topical steroids and food elimination diet for the treatment of eosinophilic esophagitis. Dig Dis Sci. https://doi.org/10.1007/s10620-018-4931-9.

4. Colson D, Kalach N, Soulaines P, et al. The impact of dietary therapy on clinical and biologic parameters of pediatric patients with eosinophilic esophagitis. J Allergy Clin Immunol Pract. 2014;2:587-593. https://doi.org/10.1016/j.jaip.2014.05.012.

5. Chehade M, Aceves SS, Furuta GT, Fleischer DM. Food allergy and eosinophilic esophagitis: what do we do? J Allergy Clin Immunol Pract. 2015;3:25-32. https://doi.org/10.1016/j. jaip.2014.11.009.

6. Liacouras CA, Furuta GT, Hirano I, et al. Eosinophilic esophagitis: updated consensus recommendations for children and adults. J Allergy Clin Immunol. 2011;128:3-20.

7. Kagalwalla AF, Wechsler JB, Amsden K, et al. Efficacy of a 4-food elimination diet for children with eosinophilic esophagitis. Clin Gastroenterol Hepatol. 2017;15:1698-1707.e7. https://doi. org/10.1016/j.cgh.2017.05.048.

8. Kagalwalla AF, Sentongo TA, Ritz S, et al. Effect of six-food elimination diet on clinical and histologic outcomes in eosinophilic esophagitis. Clin Gastroenterol Hepatol. 2006;4:1097-1102.

9. Warners MJ, Vlieg-Boerstra BJ, Bredenoord AJ. Elimination and elemental diet therapy in eosinophilic oesophagitis. Best Pract Res Clin Gastroenterol. 2015;29:793-803. https://doi.org/10.1016/j. bpg.2015.06.013.10.

10. Lynch MK, Avis KT, Dimmitt RA, Goodin BR. Topical review: eosinophilic esophagitis in children: implications for healthrelated quality of life and potential avenues for future research. $J$ Pediatr Psychol. 2015;40:727-732. 